\title{
The Value of Categorical Polythetic Diagnoses in Psychiatry
}

Sam Fellowes

Forthcoming in the British Journal for the Philosophy of Science

Some critics argue that the type of psychiatric diagnosis found in the DSM and ICD are superfluous and should be abandoned. These are known as categorical polythetic psychiatric diagnoses. To receive a categorical polythetic psychiatric diagnosis an individual need only exhibit some, rather than all, of the symptoms on the diagnostic criteria. Consequently, categorical polythetic psychiatric diagnoses only associate an individual with a range of symptoms rather than specify which symptoms they have. Drawing upon Ronald Giere's account of scientific models, I portray categorical polythetic psychiatric diagnoses as abstract models which guide the building of less abstract models. These models can specify which symptoms a particular individual has. Additionally, categorical polythetic psychiatric diagnoses can guide investigation of symptoms towards difficult to spot symptoms, guide investigation towards changing symptoms and guide investigation towards how symptoms manifest. These important roles mean categorical polythetic psychiatric diagnoses should not be abandoned.

\section{Introduction}

2 Categorical Polythetic Psychiatric Diagnoses

3 Ronald Giere's Philosophy Of Science

4 Psychiatric Diagnoses Are Not Superfluous

4.1 Advantage one: Categorical psychiatric polythetic diagnoses can 
assist accurately detecting symptoms

4.2 Advantage two: Categorical polythetic psychiatric diagnoses can assist with detecting changing symptoms

4.3 Advantage three: Categorical polythetic psychiatric diagnoses can associate symptoms with more specific behaviour

5 Broadening The Argument Beyond ASD

6 The Problem Of Flawed Categorical Polythetic Psychiatric Diagnosis

7 People Are Not Their Categorical Polythetic Psychiatric Diagnoses

8 Conclusion

\section{Introduction}

Psychiatric diagnoses made using the main diagnostic manuals, the DSM (Diagnostic and Statistical Manual) and the ICD (International Classification of Disease) are examples of categorical polythetic psychiatric diagnoses. These manuals classify people into categories; an individual either meets or fails to meet a particular set of diagnostic criteria. There is a long history of scepticism over categorical psychiatric diagnoses (as opposed to other types of psychiatric diagnosis, such as dimensional or psychodynamic diagnoses). One particular concern of critics is that categorical psychiatric diagnoses, or at least some specific categorical psychiatric diagnoses, are superfluous. In many cases, to receive a categorical psychiatric diagnosis an individual only needs to exhibit some rather than all the symptoms listed in the diagnostic criteria. Their diagnostic criteria is polythetic, meaning that there is more than one way an individual can meet the diagnostic criteria and thus qualify for that categorical polythetic psychiatric diagnosis. For example, a diagnostic criteria might require five symptoms from a list of nine and so two different people can meet that diagnostic criteria despite only having one shared symptom. This means that people with the same categorical polythetic psychiatric diagnosis can have quite different symptoms, and so knowing what 
categorical polythetic psychiatric diagnosis someone has been assigned only gives a poor indication of what symptoms they have. Those who want to know what symptoms a person with a categorical polythetic diagnosis actually manifests will need to take additional steps to find out; they will need to ask that person or observe them. This leads critics to see categorical polythetic psychiatric diagnoses as contributing nothing that cannot be established, with much greater accuracy, through directly learning what symptoms an individual exhibits. It is, of course, possible to learn both which categorical polythetic psychiatric diagnosis someone has and which symptoms they have but this seems to leave categorical polythetic psychiatric diagnoses as superfluous (Boyle [1990], p.83; Cromby et al [2015], p.116; Johnstone [2018], p.39; Kinderman et al. [2013], p.3; Latif [2016], p.290; Runswick-Cole [2016], p.27; Vanheule [2017], p.85). I respond to the claim that categorical polythetic psychiatric diagnoses are superfluous because they do not reveal which symptoms an individual exhibits. I will argue that categorical polythetic psychiatric diagnoses can make a positive contribution to understanding which symptoms someone exhibits and make a contribution to understanding how they exhibit those symptoms. ${ }^{1}$ For convenience, I shall use the acronym CPPD to refer to categorical polythetic psychiatric diagnoses.

I shall frame CPPDs in terms of Ronald Giere's account of scientific models ([1994]; [ 2004]; [2010]). Giere is a philosopher of science who has pioneered an account of scientific theories and models which accommodates their abstract nature. He shows how scientific theories are abstract. Newton's laws, for example, do not accommodate factors like wind speed, friction and shape. Giere outlines how scientific theories lack this specific detail but guide building more specific models

1 The word 'diagnosis' gets used in slightly different ways by different mental health researchers and professionals. Some hold quite a broad understanding of diagnosis, and would consider a 'diagnosis' to include a full consideration of psychological processes and life problems. There are also dimensional diagnoses whereby someone is diagnosed as having one or more attribute to a particular degree. Superfluity is considered a problem of categorical polythetic psychiatric diagnoses rather than other approaches to psychiatric diagnosis. Consequently, the sole type of diagnosis I focus upon in this article is categorical polythetic psychiatric diagnosis. 
which accommodate this detail. Following this, I will present an account according to which CPPDs operate by (i) abstracting away from details regarding the symptoms of actual diagnosed people, and (ii) can be used to guide the development of more specific models that would incorporate these details. The function that CPPDs play in model construction means they can facilitate understanding what behaviour and symptoms an individual exhibits. This means CPPDs play an important role and therefore should not be abandoned. Other authors have previously employed Giere's philosophy of science in thinking about psychiatry (Heinrichs [2015]; Murphy [2006]) and non-psychiatric medicine (Simon [2008]). However, the notion that CPPDs can guide symptom attribution has not been developed previously.

A critic might believe all possible CPPDs are superfluous, or they might believe that all currently employed (but potentially not alternative) CPPDs are superfluous, or they might focus upon claiming particular CPPDs are superfluous. I will argue that the degree to which any particular CPPD is either superfluous or non-superfluous will be case specific. In this paper I shall illustrate my arguments through considering the CPPD Autism Spectrum Disorders (henceforth ASD). ${ }^{2}$ I show that, in contrast to critics who target ASD (Timini et al. [2011], p.1; Hassal [2016], p.51; Latif [2016], p.290; Runswick-Cole [2016], p.27), it is not a superfluous CPPD. I return later in this paper to consider the ways in which ASD functions like and unlike other CPPDs. I suggest that the conclusions I draw from analysing ASD will be generalisable to a significant number of, though not all, CPPDs.

I start, in section 2, by outlining the concerns that CPPDs are superfluous. In section 3 I outline Giere's account of scientific models. I then use his account, in section 4, to show how CPPDs can provide guidance which enhances understanding of symptoms in three different helpful ways.

2 The CPPDs which were previously referred to as autism and Asperger's syndrome are called ASD since the latest edition of the Diagnostic and Statistical Manual (DSM-5). 


\section{Categorical Polythetic Psychiatric Diagnoses}

At its most basic a CPPD (as found in the DSM and ICD) is a name given to a cluster of symptoms which can occur together in an individual. For each CPPD, the DSM and ICD provides a set of diagnostic criteria which must be met to receive that CPPD. This typically consists of one or more lists of symptoms. An individual must exhibit a certain number of symptoms from each list to receive the CPPD. For example, the diagnostic criteria for ASD in DSM-5 consists of two lists. The first list covers abnormal social communication and interaction, including both verbal and non-verbal communications. The second list covers repetitive behaviour, interests and activities, including rigid thinking and hyperactive or hyporeactive sensory input (APA [2013], pp.50-1). An individual must exhibit a number of symptoms from each list to be diagnosed with ASD. The diagnostic criteria sometimes also have exclusion criteria which specify whether that CPPD should be given to an individual who also meets the diagnostic criteria of certain other CPPDs (for example, a diagnosis of ASD and schizophrenia cannot be given to the same individual even though both have some similarity of symptoms such as social impairments and unusual interests (APA [2013], p.58)).

There are various potential advantages to CPPDs. Firstly, CPPDs demarcate people into various groups which have associated clinical pictures. This can help practitioners communicate about patients, it can help other people understand the person with the CPPD and can increase the self understanding of that individual (Maung [2019], p.510; Sadler [2005], p.66). Secondly, CPPDs can be used to inform decisions over who is eligible for treatment, support, benefits and participation in research studies (Helzer et al. [2006], p.1673; Hudziak et al. [2007], p.21). Thirdly, CPPDs can be correlated with wider factors like treatment responses, gender ratios, family history and causal factors (Heinrichs, [2015]; Sadler [2005], p.66). Fourthly, CPPDs can form the basis of a cultural and political 
movement (Orgota [2013], p.80). However, all these advantages are stronger when people receiving a particular CPPD are significantly homogeneous with respect to symptoms. As I now show, critics question the merits of people with significantly different symptoms receiving the same CPPD.

Unlike dimensional diagnoses, CPPDs are binary; a particular individual either meets the diagnostic criteria or does not meet the diagnostic criteria. Everyone who is accurately assessed will fall into one of two camps: either they qualify for a particular CPPD or they do not. Under a categorical system no one only partly has a CPPD. They are also polythetic. It is in the nature of polythetic diagnostic criteria that there is more than one way to meet the diagnostic criteria. It is not the case that an individual need exhibit all of the symptoms on the diagnostic checklist to receive a CPPD. Rather, they only need exhibit a certain number of symptoms. Two individuals can thus receive the same CPPD despite not exhibiting the same symptoms (Sadler [2005], p.65; Vanheule [2017], p.85). CPPDs by default cover a heterogeneous symptom profile. Whilst many diagnoses in other branches of medicine can also cover heterogeneous symptoms, critics regard the heterogeneity of CPPDs as particularly problematic. In some cases, individuals who receive the same CPPD seem to be extremely heterogeneous. For example, there are approximately one hundred and twenty six ways to meet the diagnostic criteria for Borderline Personality Disorder (Lenzenweger [2010], p.196). ASD (the CPPD I will primarily employ as an example) similarly covers individuals who exhibit heterogeneous symptom profiles (Timini et al. [2011], p.178). Additionally, most CPPDs appear to cover individuals with significantly greater causal heterogeneity compared to diagnoses in most other branches of medicine (Cuthbert \& Insel [2013], p.3).

The polythetic nature of CPPDs means that they give clues as to what symptoms an individual might have but do not reveal what symptoms they actually do have. This is because an individual usually needs exhibit only some, rather than 
all, symptoms listed in the diagnostic criteria to receive a CPPD. Consequently, knowing an individual has a CPPD only reveals that they have some symptoms associated with their CPPD but does not reveal which ones. As a partial exception some CPPDs have a diagnostic criteria which is polythetic but still require some specific symptom to be present (such as the symptom of depressed mood being required for a diagnosis of the CPPD DSM-5 depression). However, the diagnostic criteria of many CPPDs do not have any required symptoms and those that do typically have only a small number of required symptoms. Simply knowing that someone has a CPPD results in a significant lack of specificity over which symptoms an individual with a CPPD actually has. Two quotes highlight this problem. Kinderman Read, Moncrieff and Bentall write that:

two people with a diagnosis of 'schizophrenia' or 'personality disorder' may possess no two symptoms in common, [so] it is difficult to see what communicative benefit is served by using these diagnoses. Surely a description of a person's real problems would suffice? A description of an individual's actual problems would provide more information and be of greater communicative value than a diagnostic label (Kinderman et al. [2013], p.3; see also Johnstone [2018], p.39).

Similarly, Vanheule writes that '[in categorical polythetic psychiatric diagnoses] diversity is subsumed under a general nominator and as a result the more specific features that characterize individual cases fade into an abyss of irrelevance' (Vanheule [2017], p.85). In relation to ASD, a child psychiatrist questions if "this label of 'ASD' adds anything to our understanding' (Latif [2016], p.290) and an academic and mother of an autistic child writes that she 'wonder[ed] what that word [autism] adds to the conversation' (Runswick-Cole [2016], p.27; see also Hassal 2016, p.51; Timini et al. 2011, p.1). These critics of CPPDs suggest that they are superfluous because they lack specificity over which symptoms are present.

As many critics point out, there are alternative means of revealing what 
symptoms are present and these alternatives avoid the lack of specificity. At the most basic level, upon meeting an individual with a CPPD one can directly establish which symptoms they have through directly observing them or talking to them (if they cannot talk then one might instead talk to a family member, a friend or their carer). Similarly, mental health workers could communicate about a patient or client not through discussing their CPPD but instead through discussing their symptoms. As Kinderman writes, '[a]n alternative to psychiatric diagnosis would be simply to list a person's experiences in their [the person's] own terms' ([2019], p.185). Additionally, a specialist technique called a 'formulation' (otherwise known as a psychological formulation, psychosocial formulation or a clinical case formulation) has been developed to assist psychologists and psychiatrists in establishing this and other relevant information. A formulation is the

\footnotetext{
process of co-constructing a hypothesis or 'best guess' about the origins of a person's difficulties in the context of their relationships, social circumstances, life events, and the sense that they have made of them... Formulation draws on two equally important sources of evidence: the clinician brings knowledge derived from theory, research, and clinical experience, while the service user brings expertise about their own life and the meaning and impact of their relationships and circumstances (Johnstone [2018], p.32; see also Vanheule [2017], p.172)
}

Each formulation is tailored to the specific individual (Aveline [1999], p.207; Johnstone [2018], p.32; Vanheule [2017], p.104). A formulation will aim to describe the specific behaviours an individual exhibits and describe how they feel. Unlike CPPDs, a formulation will actually describe which symptoms an individual exhibits. This means they avoid the lack of specificity of CPPDs. Additionally, a formulation aims to give an account of why an individual acts in unusual ways or feels distress. A formulation places emphasis on factors that are particular to an individual such as life history, social circumstances, life events and the particular 
psychology of the individual (Johnstone [2018], p.33; Vanheule [2017], p.172). The greater level of precision supplied by formulations compared to CPPDs is also useful when making decisions over treatment, making decisions over therapy or conducting research studies. Critics of ASD as a label (whether discussing formulations or not) have similarly suggested that simply focusing upon the individual person, rather than giving them a CPPD, would be much more useful (Hodge [2016], p.197; Latif [2016], p.297; Runswick Cole [2016], p.27).

Formulations can be used alongside CPPDs (Lingiardi et al. [2015], p.107). The DSM and the ICD recommend using formulations alongside categories (although, as critics bemoan, the DSM-5 only has two paragraphs discussing formulations (Vanheule [2017], p.153)). Additionally, a diagnostic manual has been designed to help provide formulations alongside CPPDs (Lingiardi et al. [2015]). Although I will argue for the importance of employing CPPDs I will also show that CPPDs alone are insufficient; they need supplementing with formulations. However, many advocates of formulations argue that they should be used instead of rather than alongside CPPDs. This is because CPPDs are seen as superfluous. Johnstone writes that

if a psychosocial formulation can provide a reasonably complete explanation for the experiences that have led to a psychiatric diagnosis, what ever they may be, then there is no place or need for a competing hypothesis that says 'and by the way, it is also because she has schizophrenia.' The diagnosis becomes redundant (Johnstone [2018], p.39).

Advocates of solely using formulations argue that the formulation renders the CPPDs superfluous, obsolete and irrelevant. If a formulation describes what symptoms someone has then it does everything that the CPPD is doing (and a lot more besides) making it unclear what the CPPD is contributing above and beyond what the formulation contributes (Johnstone [2018], p.39; Kinderman et al. [2013], p.3; Vanheule [2017], p.85). Critics of ASD as a label have similarly argued that 
people can be understood independently of knowing they have ASD and that ASD adds nothing to understanding them (Latif [2016], p.290; see also Hassal [2016], p.51; Timini et al. [2011], p.1; Runswick-Cole [2016], p.27).

I have now identified the argument which I respond to. Some critics see all possible, all currently employed or particular CPPDs as superfluous. They argue we should dispense with the CPPD and instead directly investigate the individual. In the remainder of this article I will argue that some CPPDs are not superfluous because they can make a positive contribution to understanding which symptoms an individual exhibits, and how they exhibit those symptoms, and that this contribution is lacking when dispensing with the CPPD to directly investigate the individual. I highlight this by focusing upon ASD and later consider if my argument can be generalised to other CPPDs.

Before I do so, however, a note regarding the scope of this paper is required. There are multiple potential concerns with the CPPDs listed in the DSM and ICD which I will not address here. Firstly, virtually no DSM and ICD CPPDs have established causal origins and are only very weakly correlated with any particular causal factor (Cuthbert \& Insel [2013], p.3; Poland [2014], p.34). Secondly, DSM and ICD CPPDs are often conceptualised as the product of biological abnormalities, but this ignores the role of the environment in either being the cause of the difficulties or in exasperating the difficulties (Johnstone [2018], p.33; Kinderman et al. [2013], p.2). Thirdly, by being binary (either present or absent), DSM and ICD CPPDs do not describe the degree to which someone meets, or fails to meet, the diagnostic criteria (Helzer et al. [2006], p.1673; Hudziak [2007], p.21). Fourthly, critics often see DSM and ICD CPPDs as having a negative impact upon the self perception of an individual with a CPPD and on the perception of others towards individuals with CPPDs (Johnstone [2014], p.275; Vanheule [2017], p.275). Fifthly, DSM and ICD CPPDs are of limited use for selecting treatment. Most drugs and therapies are not effective for everyone with a 
particular CPPD (Cooper [2007], p.150). Sixthly, DSM and ICD CPPDs are of limited use as a basis for researching causes because they typically group together causally heterogeneous individuals (Cuthbert \& Insel [2009], p.989; Poland [2014], p.46). In this paper I do not attempt to deal with such concerns.

In the next section I outline Giere's philosophy of science, which I will then use in developing my argument that CPPDs play a useful role.

\section{Ronald Giere's Philosophy Of Science}

Ronald Giere is a contemporary philosopher of science who has pioneered a modelbased account of how scientific theories relate to the world. In this section I outline his account and then apply it to psychiatry. Giere's account involves three different elements, namely physical systems, theories and models. I shall outline what these are and how they relate to one another.

A physical system is a part of the world that scientists (or indeed anyone) can investigate. A physical system may be a static state or an unfolding process. It might cover a single entity or might cover interactions between multiple entities. Giere gives the example of a swinging pendulum. At any particular moment the pendulum is at a particular height, is travelling at a particular velocity, and is taking a particular straight or circular path. These all relate to aspects of the world at a particular time.

A scientist who wished to predict height, velocity or path of a pendulum would have a number of tools at their disposal. Typically, most people see science as involving theory and data. On this understanding, height, velocity and path relate to data and these can be predicted by theory. This understanding conceals two different elements which Giere's account brings out. Most people likely presume that scientists would employ a law of nature, such as one of Newton's laws, to make these predictions. However, as Giere argues, theories like Newton's laws are of limited application for such purposes. These describe relationships between a few 
factors but do not describe many factors relevant to the actual physical system such as wind speed, friction and shape. As a consequence, scientific theories such as Newton's laws do not directly relate to the world. The theory does not describe the physical system in any concrete sense. Rather, the theory describes what would occur in a physical system if significant aspects of the actual physical system were not present.

If theories are too abstract to account for physical systems, then how do scientists go about making predictions? This is where the third aspect of Giere's account comes in. Giere outlines how scientists employ less abstract models which accommodate greater levels of specific detail that is missing from theories. Through adding specific 'conditions and additional constraints' to Newton's laws of motions 'one can generate families of representational models that can be used to represent things in the world' ([2010], p.270). He writes that,

adding the condition that $F=-k x$ yields a general model for a simple harmonic oscillator, where $x$ is the displacement from an equilibrium position [...] This model could be applied, for example, to a pendulum with a small amplitude, a mass hanging from a spring, the end of a cantilevered beam, or a diatomic molecule (Giere [2004], p.745).

Various parameters of the linear harmonic oscillator can be made more specific in various ways depending on whether it is applied to a pendulum, a spring, etc. These more specific models cover detail which is missing from the theory and is relevant to the physical system. Consequently, they are better at portraying the physical system than theories are.

Having outlined physical systems, models and theories, as employed in Giere's account I now outline two important relationships between them. Firstly, one might wonder what the point of employing theories is given that models are much more relevant to actual physical systems. Though theories do not directly relate to physical systems Giere believes theories have an important role. Giere sees theories 
as general principles which guide the building of more specific models. He writes that theories 'function more like recipes for constructing models' ([1994], p.293). Elsewhere he calls them 'general templates' ([2004], p.74). He describes how very abstract theories provide a set of general principles to which scientists can add more specific detail when creating models. Though theories are far removed from physical systems this guidance aspect means theories are not superfluous.

Secondly, models have greater resemblance to physical systems than theories do but they also do not fully resemble physical systems. Giere outlines how models do not convey all the specific details of an actual physical system. Some models have a high level of generality to them because they are intended to cover quite a lot of different things. A linear harmonic oscillator contains more specific detail than is present in Newton's laws of motion but a linear harmonic oscillator can be applied to multiple things such as pendulums, springs and cantilevered beams. Additionally, employing a model of one of those things, rather than the linear harmonic oscillator, does not specify the length of a specific spring or the specific quantity of mass. Finally, if a specific mass and spring were specified a model would still only be accurate to a certain degree rather than fully resembling an actual physical system. Experiments have a margin of error because it is typically impossible to fully shield off all interfering causal factors whilst there is always the possibility that there are unknown causal factors present (Giere [2010], p.273). Consequently, rather than seeing models as fully representing physical systems they should instead be seen as having degrees of similarity to physical systems.

I suggest that Giere's account can be applied to psychiatry. Firstly, individual people should be thought of as physical systems. They are a part of the world which has specific differences to any other part of the world. At any particular time they can be acting, perceiving, thinking and feeling in a certain way. People are parts of the world which mental health professionals (or anyone) may be 
interested in describing and understanding. People are not simply their disorder or their CPPD. People have many different attributes such as personalities, past histories, social situations and life goals, whereas a disorder (say, an underlying biological mechanism) or a CPPD at most only covers some of those attributes. ${ }^{3}$ Secondly, CPPDs should be seen as very general models which have high levels of abstraction. They are somewhat analogous to Giere's account of theories since they are abstract models which do not directly relate to the world since they do not specify which specific symptom a particular individual exhibits. ${ }^{4}$ On Giere's account the distinction between theories and models is one of degree rather than one of kind. Theories stereotypically have high levels of abstraction whereas models stereotypically have lower levels of abstraction but Giere otherwise places no firm boundaries between theories and models (see [2006], p.67). Since CPPDs are not stereotypically described as 'theories' I will simply describe them as models. Thirdly, CPPDs need be modelled onto specific individuals to attain specificity over which symptoms are present. In contrast to situations where it is only known which CPPD someone has, a CPPD can be modelled onto a specific individual by specifying which symptoms of the CPPD are present. When considered through this model the individual is no longer thought of as a general autistic person who may have a range of symptoms but instead as a specific autistic person with specific symptoms. In the same way that Giere thinks models do not fully describe physical systems this model will also not fully describe the specific individual as it only covers some of their characteristics. A description of the specific symptoms they

3 Non-physicalists might dislike describing thoughts and feelings as physical. Providing it is believed that thoughts and feelings are amenable to description and understanding then substituting 'physical system' with 'mental system' would work equally well on Giere's account.

4 CPPDs are not fully analogous to theories because Giere's put theories at a top of a hierarchy of models. In contrast, CPPD might not be put at the top of hierarchy in psychiatry. Perhaps theorising about causality, or theorising about causality of a particular CPPD, might be considered higher in the hierarchy. Such theorising might be even more abstract and distanced from the world than are CPPDs. Rather than try and establish exactly where CPPDs lie in a hierarchy of models, it suffices for my argument here that CPPDs are understood as being very abstract models which can provide guidance towards building less abstract models 
have will not reveal aspects which are not symptoms such as life goals or socioeconomic status, as well as aspects which are not psychiatrically relevant like shoe size or hair colour. The assigned symptoms will themselves also be models because they give a description of possible behaviour which is more abstract than actual behaviour (in that the symptom of low social skills can be manifested in many different ways in real world behaviour. I return to this point in section 4.3).

Giere's account emphasises the abstract and hierarchical nature of science. Scientific models vary in their degree of abstraction. This variance in abstraction may relate to a hierarchy of models. A less abstract model may be an applied version, with more real world details, of a more abstract model. CPPDs similarly exhibit these attributes of abstractness and hierarchy. By itself a CPPD is very abstract since it does not specify which symptoms an individual exhibits. However, as I have described, it can be modelled onto a specific individual to specify which symptoms are present. This more detailed model will provide more details but will not describe all aspects of the individual. Thus, like on Giere's account, there are more abstract CPPDs which lack real world detail and then, in a hierarchical relationship, a less abstract model consisting of the CPPD and a specification of an individual's specific symptoms. This more detailed model contains more real world details but still does not fully describe the person. Giere sees theories as being principles for constructing less abstract models. In what follows I will argue that CPPDs can be used to assist creating less abstract models which do specify which symptoms are present. This will show that CPPDs are not superfluous.

Giere sees his position (in his wider work on scientific perspectivism ([2006], p.3)) as falling between traditional scientific realism and anti-realism. Theories do not directly relate to the world whereas models have greater resemblance to the world but do not fully account for all aspects of physical systems. Rather than a hard scientific realist account whereby a theory corresponds with the world, Giere employs a minimal, non-metaphysical account of scientific 
realism whereby there are degrees of similarity between a theory or model and an aspect of the world ([2006], p.66). This minimal notion of realism is, I believe, the strongest possible realist account of CPPDs. They do not accurately describe actual people. They, do, however have degrees of resemblance to actual people but will not cover every aspect of the individual. In this regard there is a level of similarity between the CPPD and the actual person and then a higher level of similarity between a CPPD which has been modelled to that person (and thereby specifies which symptoms are present) and that actual person. On Giere's account abstractness is not a barrier to a minimal understanding of scientific realism and so, on this approach, a type of realism, albeit a very minimal type, is potentially applicable to CPPDs. Their abstract nature alone is not grounds for considering them as mere constructs or labels.

Philosopher of psychiatry Dominic Murphy has also related Giere's approach to psychiatric diagnoses in outlining his view that psychiatric diagnoses can be understood as exemplars. He describes exemplars as 'a representation of the clinical features and typical course of a disorder, abstracted away from the detail of individual variation' ([2006], p.202). These are abstract since Murphy believes that 'it is possible that no actual patients embody all the features of an exemplar, although patients who share a diagnosis embody at least some features of the exemplar' ([2016], pp.202-3). Like me, Murphy outlines how psychiatry has multiple models which have varying levels of abstraction. My focus here, however, differs from Murphy's. He is primarily interested in the abstract nature of exemplars and their relationship to causes. He does not discuss how psychiatric diagnoses can be used to guide detecting and understanding symptoms. I employ a model-based view of CPPDs to show that there are advantages to employing CPPDs. I will now show how CPPDs can contribute to accurately detecting and understanding symptoms. 


\section{Psychiatric Diagnoses Are Not Superfluous}

\subsection{Advantage one: Categorical psychiatric polythetic diagnoses can assist accurately detecting symptoms}

Symptoms can be difficult to accurately detect for a number of reasons. I shall show why this is and then show how CPPDs can make detecting symptoms easier by providing helpful guidance.

First, and most obviously, some symptoms are intrinsically hard to observe, or are completely unobservable by third-parties. For example, the repetitive behaviour associated with ASD is typically comparatively easy to observe compared to the repetitive thoughts since these latter symptoms are not typically observable to third-parties. Behaviour varies in how observable it is, but some behaviour has significant distance from perception.

Second, some symptoms are in principle observable but are hard to notice because they lack saliency for a variety of reasons. Many symptoms do not differ from typical behaviour qualitatively but only to a matter of degree. For example, ASD is associated with low eye contact but people with ASD typically still look others in the eyes sometimes, just to a lesser extent than do typical people. A behaviour which is rarely presented rather than absent is less likely to stand out as unusual. Sometimes an individual may have a propensity to exhibit a particular behaviour but the propensity is hidden because they consciously or unconsciously mask it (Hull et al. [2017], p.2525; Livingston \& Happé [2017], p.732). For example, an autistic individual might draw upon past experience to establish which sorts of comments are considered acceptable. They may attempt to pass as 'normal' in social situations by sticking to acceptable comments but this does not actually mean they now have typical social understanding. Symptoms can also pass unnoticed in cases where an individual has become used to behaving as they do. When a symptom becomes second nature, the affected individual, and those around 
them, might not realise it is abnormal. Some symptoms are only displayed in particular situations. For example, an individual with low social skills might socialise at normal levels around their family. The low social skills might not be noticed or not considered representative.

Third, even when a symptom is noticed it can be unclear how it should best be described. For example, imagine an individual who is considered to exhibit symptoms of anxiety. Now imagine further causal investigation established that the anxiety primarily occurs because the individual struggles to adapt to changes at work. The individual prefers to know in advance what is expected of them and becomes anxious when required to do unexpected tasks or if required to go about doing expected tasks in unexpected ways. Describing this individual as suffering from the symptom anxiety is not wholly inaccurate, but an alternative symptom can be assigned which accommodates some of this causal information. People with ASD often struggle with unexpected changes. This is a subtle symptom where the mind keeps going in the direction of what was expected and inflexibly comes up against, or struggles to accommodate to, what is unexpected. Describing the individual as suffering from the symptom of 'disliking change' better describes their issues than does the description 'anxiety'.

I suggest that CPPDs can provide guidance which can help mental health professionals to accurately detect and describe symptoms. Understood in Giere's framework, a CPPD is not in itself a particularly good model for any particular individual. Without further modelling a CPPD fails to accurately describe people, in an analogous way to how theories in the physical sciences fail to reflect actual physical systems. However, CPPDs can guide building less abstract models of people that can specify which symptoms are present, in an analogous manner to how theories in the physical sciences can guide building models which greater resemble the real world.

CPPDs are constructed from studying (in clinical or experimental settings) a 
large number of people. This process eventually results in producing a diagnostic checklist which consists of various symptoms. The main reason why any particular symptom is placed upon the checklist is that clinical or experimental evidence suggests it occurs together with other symptoms with a degree of frequency. Imagine a particular symptom more frequently occurs together with a second symptom than it does with a third. In principle, the first and second symptoms are more likely to be present on the diagnostic checklist than are the first and the third. More realistically, the presence of multiple occurring symptoms together with significant frequency is required for them to be included within a diagnostic criteria. Correlations established from clinical or experimental settings are parsed up through clinical wisdom or formal methods like factor analysis into a set of diagnostic criteria (arguably, this process will be driven by further explicit or implicit theoretical assumptions (Cooper [2007], p.51; Murphy [2006], p.226) and this process could result in many different possible sets of CPPDs, containing different CPPDs, rather than simply entailing one set of CPPDs (Kendler et al. [2011], p.1149; Kincaid [2014], p.151)). ${ }^{5}$ This means that the diagnostic criteria of a CPPD is built from studies of particular individuals. The CPPD delineates an abstract picture of what those individuals look like which can then be used to give an indication of what someone else who meets the diagnostic criteria for that CPPD looks like.

To take an example of how a CPPD can guide detecting symptoms, imagine that an individual who has not been given a CPPD meets a psychiatrist for the first time and, during the assessment, the psychiatrist notices the sole symptom of low social skills. The psychiatrist could establish which CPPDs have diagnostic criteria which include this symptom. Since CPPDs describe correlations of

5 There are multiple possible reasons why symptoms correlate together. They may be one, or multiple, underlying causes, there may be both internal and external causes and also the symptoms may be in a causal relationship with one another whereby one symptom produces another symptom. For my account here it does not matter exactly why symptoms correlate together. The mere fact that symptoms do tend to correlate suffices for CPPDs to facilitate symptom-detection. 
symptoms the psychiatrist could proceed to investigate for all the symptoms associated with all those CPPDs. Of course, low social skills occur in the diagnostic criteria of many different CPPDs, so here the guidance aspect is not particularly strong. It becomes much stronger when multiple symptoms are considered. Low social skills are correlated with many symptoms, whereas the simultaneous presence of low social skills and low eye contact is correlated with fewer. Even fewer CPPDs are covered by the simultaneous presence of low social skills, low eye contact and repetitive behaviours. Whilst not present in most CPPDs these symptoms do occur together in the diagnostic criteria for ASD. Keeping the diagnostic criteria for ASD in mind can help guide observation towards other symptoms an individual may exhibit, including difficult to spot symptoms. For example, upon establishing an individual displays repetitive behaviour there is reason to investigate whether they also have repetitive thoughts. Similarly, upon anxiety being noticed as present there is reason to investigate whether it occurs following unexpected changes. This guidance aspect can also be used outside of psychiatric evaluations to assist others in noticing symptoms, such as other mental health workers, the individual with the CPPD, their family and co-workers.

Analysing the diagnostic criteria for ASD can help elucidate this process. The diagnostic criteria for ASD has multiple aspects. Firstly, there are two broad categories: Category A covering 'Persistent deficits in social communication and social interaction across multiple contexts' ([2013], p.50) and category B covering 'Restricted, repetitive patterns of behavior, interests, or activities' ([2013], p.50). The diagnostic criteria can provide guidance at this very broad level. Imagine that a psychiatrist detects multiple symptoms from Category A within a patient during an assessment. Upon consulting or remembering the DSM it should be clear that the individual partially fits the diagnostic criteria for ASD. This then gives reason to investigate for symptoms of Category B of ASD. Note that there seems no obvious initial relationship between Category A and Category B. It does not seem to be the 
case that Category A symptoms cause Category B symptoms, or that Category B symptoms are rarer minor variations on Category A symptoms. The diagnostic criteria of the CPPD provides reason to look for otherwise seemingly completely unrelated symptoms.

The diagnostic criteria of the CPPD can also provide guidance in assigning more precise symptoms. Category A consists of three further sub-categories: 1. 'Deficits in social-emotional reciprocity [...] 2 Deficits in non-verbal communicative behaviors used for social interaction [...] 3. Deficits in developing, maintaining, and understanding relationships' ([2013], p.50). These sub-categories are a more nuanced understanding of behaviour. For example, rather than simply listing a broad notion of low social understanding these sub-categories guide awareness towards the distinction between verbal and non-verbal aspects to interpersonal communication as well as the distinction between social-emotional reciprocity and understanding of social relationships. Finally, each of the seven sub-categories (including the four from Category B) are highlighted by more detailed examples. For example, deficient social-emotional reciprocity range 'from abnormal social approach and failure of normal back-and-forth conversation; to reduced sharing of interests, emotions, or affect; to failure to initiate or respond to social interactions' ([2013], p.50). All of these are again more nuanced and more subtle ways to describing autistic social skills. Upon the detection of some symptoms associated with ASD the diagnostic criteria gives reason to investigate for more precisely defined symptoms.

My claim fits well with the experience of people who have ASD. They often feel that they have a greater understanding of who they are after receiving a CPPD of ASD. For example, an autistic person who interviewed multiple autistic people writes that ' $[\mathrm{m}]$ any felt that this [being diagnosed] led to a greater sense of self-understanding' (Milton \& Sims [2017], p.152). I suggest that this is partly because the CPPD can help them notice and understand how they are acting (see 
Hacking ([1999]) and Orgota ([2013]) for additional reasons why a psychiatric diagnosis can change self-understanding).

It could be argued that well-trained experienced clinicians do not need the guidance supplied by the diagnostic criteria of CPPDs. They might be able to draw upon other resources to achieve similar effect. It is true that some symptoms will be easier to spot than others, and that some clinicians will have significantly more training and experience than others. In some context the guidance supplied by the diagnostic criteria of CPPDs may not strictly speaking be needed (though this would not necessarily show that diagnostic criteria are worthless as using them might still save time). However, it is worth remembering that many psychiatric symptoms are subtle. Also, many clinicians are not experts. A great deal of care is provided by GPs. The training of mental health professionals is also mixed. A 2014 report gives statistics for mental health professionals within the UK. Consultant psychiatrists made up $4.5 \%$ of the mental health workforce, clinical psychology staff 7.7\%, and community psychiatric nurses 48.3\% (NHS Factsheet [2016]). Finally, a recent study shows that clinicians do often explicitly consider DSM diagnostic criteria in clinical settings. First et al. ([2019]) write that 'a majority of clinicians review the DSM criteria either from memory or in written form to determine whether diagnostic criteria are met during the initial assessment' (p.160). Many clinicians do keep DSM diagnostic criteria in mind when assessing patients and I suggest there are benefits to doing so.

\subsection{Advantage two: Categorical polythetic psychiatric diagnoses can assist with detecting changing symptoms}

The symptoms an individual exhibits can change over time (Cuthbert \& Insel [2013], p.5; in relation to ASD see Walsh et al. [2011], p.606). Symptoms may start being exhibited, may stop being exhibited and may change how or when they are exhibited. In this section I will outline why this is and then outline how 
CPPDs can guide awareness towards the possibility that changes can occur and assist establishing which changes have occurred.

Whether an individual manifests a symptom and how it manifests can depend upon external and internal causes. Different symptoms will commonly be displayed in different environments. An individual may have a propensity to exhibit a symptom but will only manifest it in a certain environment, and the same symptom may manifest differently in different environments. As such, if the environment changes then so too which symptoms are exhibited. Additionally, an individual's personality and past experience can influence whether and how symptoms manifest. Most people will undergo changes to their personality throughout the course of their life. Individuals constantly gain new experiences and may change their views about their past experiences. Finally, whether and how symptoms manifest can be influenced by the stance the individual takes towards their symptoms. An individual might gain greater awareness of why a symptom occurs or which behaviours are manifestations of the symptom. They may alter whether they see the symptom as positive or negative. They may actively try and stop the symptom occurring or change how it occurs.

Given the significant possibility that the symptoms an individual manifests will change over time it is helpful to associate an individual with a wider range of symptoms than those they exhibit at any particular time. Rather than simply modelling the symptoms an individual has it is thus helpful to consider them an instance of a wider CPPD which is associated with a wider range of symptoms than any particular individual exhibits. In an analogous way to Giere's notion of a theory, a CPPD can act as a template or recipe which entails multiple, more specific models. Consequently, a CPPD does not tie the individual to one specific model, which entails one specific set of symptoms, but rather suggests a range of models may be possible which cover various sets of symptoms. Since there is more than one way to be, say, autistic, it is implicitly suggested that symptoms may 
change, and so raises awareness that change is a possibility. More importantly, a CPPD can guide awareness toward detecting which new symptoms are being exhibited. By being polythetic a CPPD associates an individual with symptoms they are not currently exhibiting but might exhibit in the future. This guides awareness towards a range of symptoms that are more likely to start being exhibited by people with that CPPD. This is helpful given the hundreds of possible symptoms which could be exhibited. Additional guidance is provided if a CPPD is associated with a developmental course, a periodic course or a deteriorating course, such as is respectively associated with $\mathrm{ASD}$, bipolar disorder, and schizophrenia. This may allow some level of predictability as to which symptoms will start occurring and which will stop occurring. None of this guidance is given when simply considering which symptoms are exhibited at a particular time.

The way that CPPDs provide guidance over detecting changing symptoms is also relevant for difficult to spot symptoms. Imagine an individual exhibited a subtle symptom and a psychiatrist employed their specialist training to detect it. Now imagine an individual only starts exhibiting the symptom after assessments by psychiatric services and that they are no longer seeing psychiatric professionals. The specialist knowledge supplied by the psychiatrist would no longer be present whereas the guidance provided by a CPPD would still be present. This would assist with symptom attribution over difficult to spot symptoms.

\subsection{Advantage three: Categorical polythetic psychiatric diagnoses can associate symptoms with more specific behaviour}

A CPPD can guide building more specific models of how symptoms manifest. Many symptoms are relatively general descriptions that cover somewhat diverse behaviours (Fellowes [2017], p.285). Giere argues that models are not the same as physical systems, rather models can only have degrees of resemblance to physical systems. Similarly, symptoms are not the same as actual instances of behaviour, 
rather, they can at best have high degrees of resemblance. For example, the symptom 'low social skills' can manifest in significantly different ways for significantly different reasons. Four examples highlight this. One individual might wish to socialise in what are typically considered to be normal ways but has a significant lack of social understanding. They misjudge social boundaries and they say things which are considered unacceptable. They have an inbuilt lack of intuitive understanding (one which they may be able to improve through practice or support but only to a limited degree). A second individual might exhibit low social skills because they are very withdrawn. Their subjective lifeworld (the way in which things appear meaningful to them) is so different to most people that they do not wish to engage on a social level. They instead withdraw into their own world. A third individual might in principle be able to socialise in a normal way. However, they generally have no desire to follow social conventions except when they are able to manipulate them to their own advantages. They have no desire for close relationships and may see themselves as above petty social conventions. A fourth individual might be capable of exhibiting normal levels of social skills providing they can remain sufficiently calm in social situations. However, the individual suffers from significant levels of anxiety in social situations. The panic which accompanies most social situations makes it difficult for them to function socially and this may cause them to act in ways deemed socially inappropriate. All these four individuals would be considered to exhibit 'low social skills' despite their differences in behaviour and different reasons for exhibiting that behaviour. This shows that symptoms are missing some of the specific detail present in instances of behaviour. Knowing that an individual has a specific symptom indicates a range of possible behaviours rather than specifies which behaviour is present.

CPPDs can guide building more specific models of symptoms which indicate a narrower range of behaviour. By itself a symptom can be associated with multiple ways of manifesting but when considered as part of a CPPD there may be 
an indication that some manifestations of the symptom are more likely than others. For example, each manifestation of low social skills I outlined above is associated with a particular CPPD. ASD is most associated with the ingrained lack of social understanding, schizophrenia with withdrawal, personality disorders with indifference, and social anxiety with anxiety. In all these cases a CPPD can guide building more specific models of how a particular symptom manifests. These more specific models mean that the symptom now gives a greater indication of which behaviour is likely to occur. The symptoms indicate which manifestation is most likely but helpfully does not disassociate the individual from less likely manifestations. Though low social skills in autism is most associated with ingrained lack of social skills an autistic individual may be withdrawn, may see themselves as above social convention and may face anxiety in social situations. The symptom allows an individual to be understood in diverse ways but by situating the symptom within a CPPD guidance is given over which way or ways is more likely. Knowing an individual both exhibits a symptom and has a CPPD may reveal more information about the behaviour associated with the symptom compared to simply knowing they exhibit the symptom.

The guidance supplied by CPPDs is lacking if clinicians dispense with CPPDs and just directly describe the individual. To make up for this deficit attempts to directly describe the individual would need a more nuanced set of symptoms than are currently employed. Plausibly, there are many advantages to formulating new, more nuanced symptoms which would each cover a more specific set of behaviours. Advocates of formulations sometimes argue currently employed symptoms are often conceptualised in too broad a manner and that more specific descriptions would usually be more helpful (Vanheule [2017], p.100). Doing this would go some way to make directly describing the individual work. However, there would be many disadvantages to formulating these more nuanced symptoms. First, this would require many more symptoms to be formulated. Psychiatrists 
would need to give a much larger number of symptoms fair consideration when diagnosing symptoms meanwhile other mental health workers and the general public would need awareness of a much greater range of symptoms. Second, even if there was awareness of all these more specific symptoms there would also have to be awareness of how they differ from one another. In a system of more nuanced symptoms it will be more common that two different symptoms cover overlapping behaviour and that some symptoms will be more specific versions of other symptoms. This can be expected to cause problems regarding the reliability of symptom attribution (whether different psychiatrists attribute different symptoms to the same individual) and will cause non-mental health professionals significant difficulties at understanding which symptoms cover which behaviour. Thirdly, assigning symptoms which are tied to very specific behaviour seems less conducive to the notion that an individual might change their behaviour across time. In contrast, formulating symptoms with a higher degree of generality means they can be associated with a greater range of behaviour and which are exhibited may be influenced by changing factors which are internal or external to the individual.

There are many possible degrees of generality at which symptoms can be formulated and I do not endorse the current degree of generality. Rather, I have highlighted how symptoms with a degree of generality can be supplemented by the guidance provided by CPPDs. This guidance aspect helps symptom attribution with currently employed symptoms and would likely still provide guidance to an alternative set of symptoms formulated at an alternative set of generality. Directly describing the individual faces the problem of either having generalised symptoms without the guidance provided from CPPDs or significantly increasing complexity by formulating many new symptoms which are each associated with more specific behaviour.

\section{Broadening The Argument Beyond ASD}


I have argued that CPPDs play a valuable role in guiding the detection and understanding of symptoms. Throughout my argument I have employed ASD as an example. It is now time to argue that the argument can be generalised to many other CPPDs although I do not claim it is generalisable to all. There may be some cases where symptom attribution is so easy that there is no need for CPPDs to provide guidance. For example, perhaps an individual repetitively banging their head against a floor seems sufficiently easy to spot regardless of any guidance supplied by CPPDs. In other cases, a particular CPPD may provide relatively little guidance. The diagnostic criteria for certain CPPDs include very few symptoms, for example trichotillomania (hair pulling) and pica (eating food which lacks nutritional value). Establishing that an individual exhibits two symptoms on the diagnostic criteria of a particular CPPD provides little guidance if the diagnostic criteria only consists of three symptoms. Perhaps in these cases CPPDs do not make much of a helpful contribution and might not be worth employing. However, very many CPPDs are associated with multiple symptoms (like ASD, schizophrenia and bipolar disorder). The argument developed here will apply to all such cases.

\section{The Problem Of Flawed Categorical Polythetic Psychiatric Diagnosis}

I now discuss a possible objection to my argument. A critic could grant that CPPDs provide guidance but then claim that the guidance is harmful. Critics often claim that CPPDs in the DSM and ICD group together symptoms in ways which fail to reflect how symptoms actually cluster together in people (Cuthbert \& Insel [2013], p.3; Kinderman et al. [2013], p.2). If so CPPDs could guide investigation towards the wrong symptoms. It could guide investigation towards symptoms which someone does not have, potentially leading to them being incorrectly assigned symptoms which they do not have. Additionally, it might fail to guide toward symptoms that someone does have.

Whilst this is a legitimate concern it is important to qualify the extent of 
this problem. Firstly, it is not a problem which is specific to CPPDs. There are general problems, arising from the theory-laden nature of evidence, which are potentially applicable to all scientific theories. A flawed theory is likely to generate a flawed model. Consequently, the possibility of being flawed is insufficient to dismiss all CPPDs. There needs be reason to believe particular CPPDs are flawed.

Secondly, critics argue that CPPDs are flawed because people with very heterogeneous symptoms can receive the same CPPD, but my argument shows that covering heterogeneous symptoms does not prevent a CPPD providing helpful guidance. I have shown how a CPPD can provide guidance even though people with the CPPD do not have all the same symptoms. Therefore, a CPPD can provide helpful guidance despite covering individuals with heterogeneous groupings of symptoms. There will be a limit to the level of heterogeneity before guidance starts being unhelpful but heterogeneity of symptom profiles does not always entail that the CPPD is useless. Consequently, critics of CPPDs do not merely need show a particular CPPD is given to people with heterogeneous symptoms but need show that the people who have received the CPPD are so heterogeneous that the CPPD no longer provides helpful guidance.

Some, however, do argue that the current DSM (and very similar ICD) are so bad that they are misleading. The Research Domain Criteria project (known as RDoC) was set up by the National Institute for Mental Health (the main U.S. government body for mental health). The $\mathrm{RDoC}$ project assumes that most currently employed CPPDs are too heterogeneous both causally and in associated symptoms. The $\mathrm{RDoC}$ project believes that the level of heterogeneity holds back causal investigation. Consequently, the $\mathrm{RDoC}$ project intends to investigate causes independently of currently employed CPPDs (Cuthbert \& Insel [2009], p.989).

The eventual hope is that the $\mathrm{RDoC}$ project will lead to a new and better nosology for psychiatry. Whether such a future nosology will still support CPPDs is unclear. RDoC literature generally favours moving towards a dimensional approach 
but whether this will be used alongside or instead of a categorical approach is rarely commented upon. Many of those who advocate for employing a dimensional approach believe that dimensional approaches should be used alongside categorical approaches (Helzer et al. [2006], p.1675; Hudziak et al. [2007], p.21). The aim of my argument is not to defend the DSM in its current form, but rather to argue against critics who claim that CPPDs are necessarily superfluous. I accept (though do not endorse) that the current nosological system might be flawed, but have shown here that seeking well grounded CPPDs is worthwhile.

If the future consists of new, superior CPPDs (enabled by RDoC or some other research programme) then the benefits of CPPDs that I argue for here will still be applicable.

\section{People Are Not Their Categorical Polythetic Psychiatric Diagnoses}

In this article I have responded to the concern that CPPDs lack specificity. I have shown previously unrecognised advantages to this lack of specificity. However, having argued for the importance of CPPDs I now explain why, on the account I have developed, they are not sufficient but need supplementing with more detailed models.

I have employed Giere's account to portray CPPDs as abstract models which are analogous to Giere's notion of scientific theories. Giere demarcates theories from models and physical systems. A model has additional detail which is not present within the theory. The physical system is the thing which is being modelled. Following this, I demarcated CPPDs, which do not specify the specific symptoms someone exhibits, from the more detailed model of a CPPD which specifies which symptoms a particular individual has. Additionally, I have demarcated both these from the actual person. CPPDs are too abstract to directly resemble specific people whilst modelling a CPPD onto a particular individual results only in degrees of resemblance to that specific person. Giere writes that 
theories 'cannot by themselves be used to make any direct claims about the world' (Giere [2010], p.270). When thought of in terms of CPPDs this acts as a clear statement that CPPDs do not resemble actual individuals. Through employing Giere's account, the framework for understanding CPPDs developed here highlights many of their limitations that other authors have also highlighted (Johnstone [2014], p.275; Lingiardi et al. [2015], p.110; McWilliams [2005], p.143; Vanheule [2017], p.275).

My argument shows that CPPDs need supplementing with models which specify which symptoms are present and with approaches which model other aspects of the individual such as their past life history, their current life situation or aspects of their personality which are not symptoms. Formulations would be one means of establishing this information. A formulation is intended to give a description of a specific person, specifying which symptoms they have and specifying many other aspects of the individual. A CPPD does not specify which symptoms are present whereas a formulation is a means of establishing a less abstract model which would specify which symptoms are present (and much else besides). Given the advantages to knowing which specific symptoms an individual exhibits there is good reason to supplement CPPDs with formulations.

\section{Conclusion}

In this paper I have responded to the argument that CPPDs are superfluous. Critics claim CPPDs are superfluous because they only associate an individual with a range of symptoms rather than specify which symptoms are present.

In response to this concern I have framed CPPDs as analogous to Giere's account of scientific theories. I portray CPPDs as abstract models which guide the building of more specific models. In doing this I have highlighted three previously unrecognised advantages to employing CPPDs. Firstly, the polythetic diagnostic criteria of CPPDs can help guide symptom attribution. Secondly, a CPPD can help 
encourage awareness that symptoms can change and can guide attention to what changes may have occurred. Thirdly, a CPPD can indicate more likely ways a symptom will manifest as a behaviour. This suggests that CPPDs have advantages which have been unrecognised by their critics. Consequently, I suggest that there is good reason to keep employing CPPDs. I have, however, acknowledged that CPPDs have significant limitations and that they should be supplemented with formulations.

\section{Acknowledgements:}

I would like to thank Rachel Cooper, Jane Pitt, George Turner, Sen Bhuvanendra and Dan Degerman who read and offered helpful criticism of this article. I am a Wellcome Trust funded Research Fellow [209868/Z/17/Z].

Sam Fellowes Politics, Philosophy and Religion University of Lancaster Lancaster, United Kingdom m.fellowes1@lancaster.ac.uk

\section{References}

APA. [2013]: Diagnostic and statistical manual of mental disorders (5th ed), Washington, DC: American Psychiatric Association.

Aveline, M. [1999]: 'The advantages of formulation over categorical diagnoses in explorative psychotherapy and psychodynamic management', The European Journal of Psychotherapy, Counselling \& Health, 2/2, pp. 199-216.

Boyle, M. [1990]. Schizophrenia: a scientific delusion?, London: Routledge.

Cromby, J., Harper, D. and Reavey, P. [2015]: Psychology, Mental Health and Distress. Basingstoke: Palgrave-McMillian.

Cooper, R. [2007]: Psychiatry and the Philosophy of Science, Stocksfield: Acumen. 
Cuthbert, B. N. and Insel, T. R. [2009]: 'Endophenotypes: Bridging Genomic Complexity and Disorder Heterogeneity', Biological Psychiatry, 66, pp. 988-989.

Cuthbert, B. N. and Insel, T. R. [2013]: 'Towards the future of psychiatric diagnosis: the seven pillars of RdoC', BMC Medicine, 11/126, pp. $1-8$.

Fellowes, S. [2017]: 'Symptom modelling can be influenced by psychiatric categories: choices for research domain criteria (RDoC)', Theoretical Medicine and Bioethics, 38/4, pp. 279-294.

First, M. B., Erlich, M. D., Adler, D. A., Leong, S., Dixon, L. B., Olsin, D. W., Goldman, B., Koh, S., Levine, B., Berlant, J. L. and Siris, S. G. [2019]: 'How the $D S M$ is used in clinical practice', The Journal of Nervous and Mental Disease, 207/3, pp. $157-161$.

Giere, R. N. [1994]: 'The Cognitive Structure of Scientific Theories', Philosophy of Science, 61/2, pp. 276-296.

Giere, R. N. [2004]: 'How models are used to represent reality', Philosophy of Science, 71/5 (Proceedings), pp. S742-S752.

Giere, R. N. [2006]: Scientific Perspectivism. Chicago: Chicago University Press.

Giere, R. [2010]: 'An agent-based conception of models and scientific representation', Synthese 172/2, pp. 269-281.

Hacking, I. [1999]: The Social Construction of What. Cambridge, Harvard University Press.

Hassall, R. [2016]: 'Does Everybody with an Autism Diagnosis Have the Same Underlying Condition', In K. Runswick-Cole., R. Mallett and S. Timini (eds), ReThinking Autism, London: Jessica Kingsley Publishing, pp. 49-66.

Helzer, J. E., Kraemer, H. C., and Krueger, R. F. [2006]: 'The feasibility and need for dimensional psychiatric diagnoses', Psychological Medicine, 32, pp. 1671-1680. Heinrichs, D. W. [2015]: 'Model-based Science and the Ethics of Ongoing Treatment Negotiation'. In: J. Z. Sadler., W. V. Staden and K. W. M. Fulford (eds), The Oxford Handbook of Psychiatric Ethics, Oxford: Oxford University Press. 
Hodge, N. [2016]: 'Schools without labels', In K. Runswick-Cole., R. Mallett and S. Timini (eds), Re-Thinking Autism, London: Jessica Kingsley Publishing, pp. 185 $-203$.

Hudziak, J. J., Achenbach, T. M., Althoff, R. R. and Pine, D. S. [2007]: 'A dimensional approach to developmental psychopathology', International Journal of Methods in Psychiatric Research, 16/S1, pp. 16-23

Hull, L., Petrides, K. V., Allison, C., Smith, P., Baron-Cohen, S., Lai, M-C. and Mandy, W. [2017]: “ “Putting on my best normal”: social camouflaging in adults with autism spectrum conditions', Journal of Autism and Developmental Disorders, 47/8, pp. $2519-2534$.

Johnstone, L. [2014]: 'Controversies and debates about formulation', In L. Johnstone and R. Dallos (eds), Formulation in Psychology and Psychotherapy: Making sense of people's problems, 2nd edition, Hove: Routledge, pp. 260-289.

Johnstone, L. [2018]: 'Psychological formulation as an Alternative to Psychiatric Diagnoses', Journal of Humanistic Psychology, 58/1, pp. 30-46.

Kendler, K. S., Zachar, P. and Craver, C. [2011]: 'What kinds of things are psychiatric disorders?', Psychological Medicine, 41, pp. 1143-1150.

Kincaid, H. [2014]: 'Defensible Natural Kinds'. In H. Kincaid and J. A. Sullivan (eds), Classifying Psychopathology, Massachusetts: MIT Press, pp. 145-174.

Kinderman, P., Reed, J., Moncrieff, J. and Bentall, R. P. [2013]: 'Drop the Language of Disorder', Evidence Based Mental Health, 16/1, pp. 1-3.

Kinderman, P. [2019]: 'From chemical imbalance to power imbalance: a manifesto for mental health'. In. W. Jo (ed), Drop the Disorder, Monmouth: PCCS Books, pp. $182-190$.

Latif, S. [2016]: 'The Ethics and Consequences of Making Autism Spectrum Disorder Diagnoses.' In K. Runswick-Cole., R. Mallett and S. Timini (eds), ReThinking Autism, London: Jessica Kingsley Publishing, pp. 288-299.

Lenzenweger, M. F. [2010]: 'Contemplations on Meehl', In T. Millon., R. F. 
Krueger and E. Simonsen (eds), Contemporary Directions in Psychopathology, Scientific Foundations of the DSM-V and ICD-11, New York: The Guilford Press, pp. $187-204$.

Lingiardi, V., McWilliams, N., Bornstein, R. F., Gazzillo, F. and Gordon, R. M. [2015]: 'The Psychodynamic 'Diagnostic Manual Version 2 (PDM-2): Assessing Patients for Improved Clinical Practice and Research', Psychoanalytic Psychology, 32/1, pp. 94-115.

Livingston, L. A. \& Happé, F. [2017]: 'Conceptualising compensation in neurodevelopmental disorders: Reflections from autism spectrum disorder', Neuroscience \& Biobehavioral Reviews, 80, pp. 729-742.

Maung, H. H. [2019]: 'The Function of Diagnoses in Medicine and Psychiatry'. In S. Tekin and R. Bluhm (eds), The Bloomsbury Companion To Philosophy of Psychiatry., London: Bloomsbury, pp. 507-526.

McWilliams, N. [2005]: 'Preserving Our Humanity as Therapists. Psychotherapy: Theory', Research, Practice, Training, 42/2, pp. 139-151.

Milton, D. E. M. and Sims, T. [2017]: 'How is a sense of well-being and belonging constructed in the accounts of autistic adult.' In Damien E. M. M. (ed), A Mismatch of Salience, Hove: Pavilion, pp. 143-162.

Murphy, D. [2006]: Psychiatry in the Scientific Image. Massachusetts: Massachusetts Institute of Technology.

NHS Factsheet. [2016]: 'Key facts and trends in mental health', avaliable at $<$ https://www.nhsconfed.org/-/media/Confederation/Files/Publications/Documents/MHNkey-facts-and-trends-factsheet Fs1356 3 WEB.pdf $>$

Orgota, F. [2013]: 'Cerebralizing Autism with the Neurodiversity Movement'. In J. Davidson and M. Orsini, (eds), Worlds of Autism, Minneapolis: University of Missesota Press, pp. 7396

Poland J. [2014]: 'Deeply Rooted Sources of Error and Bias in Psychiatric Classifications'. In H. Kincaid and J. A. Sullivan (eds), Classifying 
Psychopathology, Massachusetts: MIT Press, pp.145174. MIT Press: Massachusetts.

Runswick-Cole, K. [2016]: 'Understanding This Thing Called Autism'. In K. Runswick-Cole., R. Mallett and S. Timini (eds), Re-Thinking Autism, London: Jessica Kingsley Publishing, pp. 1929.

Sadler, J. Z. [2005]: Values and Psychiatric Diagnoses. Oxford: Oxford University Press.

Simon, J. R. [2008]: 'Constructive realism and medicine: An approach to medical ontology.' Perspectives in biology and medicine, 51/3, pp. 353366.

Timini, S., Gardner, N and McCabe, B. [2011]: The Myth of Autism. PalgraveMcMillian.

Walsh, P., Elsabbagh, M., Bolton, P. and Singh, I. [2011]: 'In search of biomarkers for autism: scientific, social and ethical challenges', Nature Reviews Neuroscience, 12, pp. 603612.

Vanheule, S. [2017]: Psychiatric Diagnosis Revisited: From DSM to Clinical Case Formulation. Cham: Palgrave Macmillan. 